\title{
An Enhanced Routing Metric for Fading Wireless Channels
}

\author{
Daniel de O. Cunha ${ }^{1,2}$, Otto Carlos M. B. Duarte ${ }^{2}$, and Guy Pujolle ${ }^{1}$ \\ ${ }^{1}$ Laboratoire d'Informatique de Paris 6 (LIP6) \\ ${ }^{2}$ Grupo de Teleinformática e Automação (GTA) \\ Université Pierre et Marie Curie - Paris VI \\ Paris, France \\ Universidade Federal do Rio de Janeiro \\ Rio de Janeiro, RJ, Brazil
}

\begin{abstract}
In this paper we propose the enhancement of routing metrics through a more complete view of the physical channel. Using cross-layer optimizations, we develop the Distribution Based Expected Transmission Count (DBETX), which improves the performance of the network in the presence of varying channels. Through observations of the wireless link, the nodes are able to estimate how the wireless link behaves. The proposed metric exploits this estimation to increase routing efficiency. The proposed metric is shown to outperform the conventional ETX metric in the presence of fading. The improvement over ETX increases with the network density because connectivity increases and more routing options become available. Results show a reduction of up to $26 \%$ in the Average Number of Transmissions per link and an increase of up to $32 \%$ in the end-to-end route availability.
\end{abstract}

\section{INTRODUCTION}

Wireless networks are popular due to their high flexibility and low deployment cost [1]. Nevertheless, the performance of these networks is closely related to the capacity of the routing protocol to select adequate routes that minimize losses and maximize throughput. This is especially true for ad hoc networks, which are not planned and randomly established.

Initially, wireless networking research was based on simplified physical models and, thus, contention and mobility represented the main factors impacting network performance [2]. Recent works, however, show that the variability of the wireless channel is an important factor and must not be neglected. Schmitz et al. [3] show that in a realistic environment, even under optimistic conditions, the influence of the wireless channel variations over the topological changes is more significant than that of the mobility. Therefore, it is necessary to develop quality-aware routing metrics that reflect the stability of the links.

Adya et al. propose the Per-Hop Round Trip Time metric [4]. This metric is based on the round trip delay experienced by probes sent to the neighbor nodes. To obtain the delays, nodes periodically send probes to their neighbors. Upon the arrival of a probe, the neighbors acknowledge the reception appending their timestamp. The sending node is thus able to calculate the delay.

The Expected Transmission Count (ETX) [5] estimates the average number of transmissions needed to send a packet through a wireless link. Nodes periodically send probe packets to their neighbors and based on the percentage of received

This work has been supported by CNPq, CAPES, FAPERJ, FINEP, RNP, FUNTTEL (Brazil), and ANR - Projet RADIC-SF (France). probe-acknowledgments nodes estimate the success probability $\left(P_{\text {suc }}\right)$ of a link. The average number of transmissions needed to transmit a packet through a specific link is $1 / E\left[P_{\text {suc }}\right]$.

Draves et al. compare the performances of the above mentioned and other metrics in multi-hop wireless networks [6]. Compared to the conventional hop-count metric and the other analyzed quality-aware metrics, the ETX metric results in longer but more efficient routes.

Although very efficient, the ETX metric is based on the average behavior of the link $\left(E\left[P_{s u c}\right]\right)$. As recent results show the importance of the physical layer properties in the optimum protocol design for wireless networks [7], the use of more complete physical layer information can further improve the performance. Koksal and Balakrishnan propose two enhancements to the ETX metric [8]. The first proposition is the Modified ETX, or mETX. Differently from the original ETX, the mETX metric is computed as $E\left[1 / P_{\text {suc }}\right]$. This modification captures better the effects of the link variation on the Expected Transmission Count, which is calculated using the function $1 / x$. The second proposed metric, the Effective Number of Transmissions - ENT, evaluates the probability that the number of transmissions exceed the maximum number of MAC-layer retransmissions. The ENT metric is then used to prune the routing graph, eliminating lossy links. Routing is accomplished using the original ETX metric.

In this paper we propose the Distribution Based Expected Transmission Count metric (DBETX). The DBETX metric is calculated based on physical level measurements and locally available information such as the noise floor and the selected modulation scheme. Based on the link measurements, the node is able to estimate the probability density function (pdf) of the experimented signal-to-noise plus interference ratio (SNIR). For a given modulation, it is possible to calculate the expected bit error rate (BER) and, as a consequence, the expected packet error rate (PER). Thus, the average number of required transmissions in a given link can be estimated based on the SNIR. The DBETX metric takes the maximum number of MAC-layer retransmissions into account in order to derive the number of required transmissions. Besides, DBETX penalizes lossy links in order to find routes with lower endto-end loss rates. Differently from the proposition of Koksal and Balakrishnan, we favor the choice of low loss-rate links, instead of pruning the network, to avoid network partition.

The rest of the paper is structured in the following way. Section II details how fading affects the channel conditions. 
In Section III we propose and explain the DBETX metric. Then, the simulations are presented in Section IV, showing the efficiency of the DBETX metric. Finally, conclusions and future research work are discussed in Section V.

\section{THE FADING CHANNEL}

In wireless communications with the presence of scatterers, the signals travel from the transmitter to the receiver through multiple paths. When there is mobility of the transmitter, receiver, or the environment, these multiple paths change, resulting in fluctuations in the amplitude and phase of the received signal.

One main factor dictating the fading behavior is the presence or not of a direct line-of-sight [9]. When there is no direct path, all the energy is received over scattered paths and the channel presents Rayleigh fading. On the other hand, when there is a strong line-of-sight component, the channel is classified as a Ricean fading channel.

The probability density function for the received power $(p)$ in a Ricean fading channel is given by [10]

$$
f_{p}(p \mid \bar{p}, K)=\frac{1+K}{\bar{p}} e^{-K} e^{\frac{p(1+K)}{\bar{p}}} I_{0}\left(\sqrt{\frac{4 K(1+K) p}{\bar{p}}}\right),
$$

where $\bar{p}$ is the average received power obtained by a large scale propagation model, $I_{0}$ is the modified bessel function of first kind and zero order, and $\mathrm{K}$ is the Ricean factor. Defining $p_{d}$ as the power in the direct path component and $\bar{p}_{s}$ as the average power in the scattered components, we have

$$
K=\frac{p_{d}}{\bar{p}_{s}} .
$$

Therefore, the higher the K-factor of a link is, the greater the influence of the direct path. The Rayleigh fading channel can be seen as a special case of the Rician channel where $K=0$, meaning that all the signal is received through scattered paths. In this case, (1) reduces to an exponential distribution and the pdf of the received power is

$$
f_{p}(p \mid \bar{p})=\frac{1}{\bar{p}} e^{\frac{p}{\bar{p}}} .
$$

Comparing (1) to (3), we can see that the Rayleigh channel is characterized by only one parameter, the average received power, whereas the Rician channel is characterized by two parameters, the average received power and the amount of energy in the direct path. Fig. 1 shows the distribution of the received power for different $\mathrm{K}$-factors and $\bar{p}=1$.

As Fig. 1 shows, averagely equal channels can exhibit different behaviors depending on the fraction of power received through the direct path. Assuming the 64-QAM modulation, 500-byte packets and deriving the maximum range based on the minimum sensibility of IEEE $802.11 \mathrm{~g}$ interfaces operating at $54 \mathrm{Mbps}$ [11], Fig. 2 shows how the existence of a line-ofsight impacts the success probability of a transmission.

As shown on Fig. 2, the amount of energy on the direct path changes significantly the average success probability of the link. For Rayleigh channels, even small distances result in a reduced success probability. As the amount of energy on the

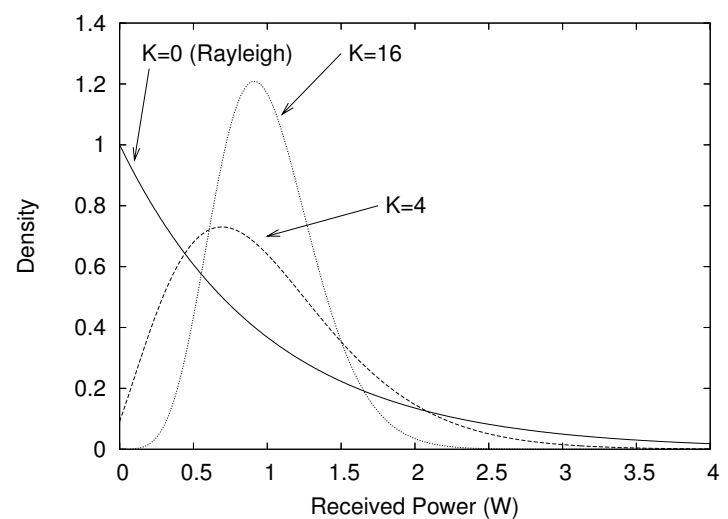

Fig. 1. Distribution of the received power of different channels.

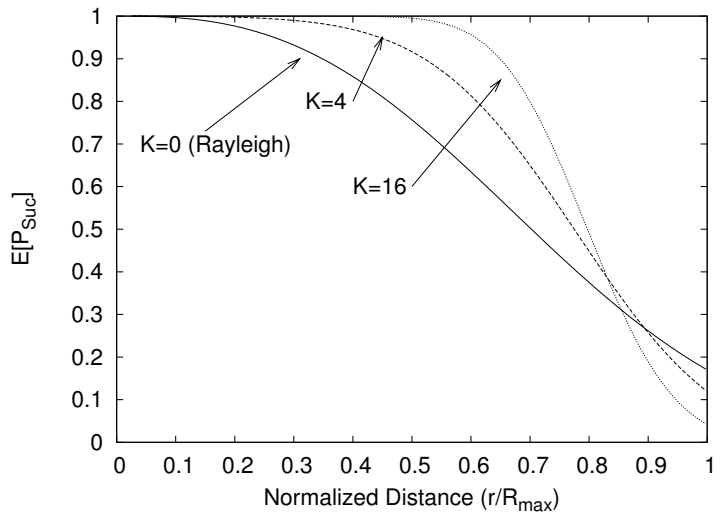

Fig. 2. Average success probability as a function of the link distance.

direct path increases, a larger distance is required to reduce the average success probability, but its reduction is sharper.

The rapidity of the signal variations is related to the carrier frequency $\left(f_{c}\right)$ and the transmitter, receiver, and environmental speed. The coherence time, $T_{0}$, is an estimation of the amount of time during which the channel response is approximately invariant. Based on the relation between the coherence time and the duration of a symbol transmission, $T_{s}$, the channel can be categorized as a fast fading or a slow fading channel. When $T_{0}<T_{s}$, the channel is said to be a fast fading channel. In this case, the channel varies during the transmission of one symbol, leading to the distortion of the baseband pulse. On the other hand, when $T_{0}>T_{s}$, the channel is classified as a slow fading channel. In this case, the primary effect of fading is the loss of SNR [12]. In this paper we consider only slow fading channels. The next section details the DBETX metric and how it takes into account the channel variations and the maximum MAC-layer retransmissions.

\section{The Proposed Metric}

In order to understand the key idea of our proposal, first consider a simplified example. Imagine we can send a packet through two different links, $a$ or $b$. Link $a$ is a very stable link, presenting always $P_{s u c}=0.5$, whereas link $b$ is a varying link, presenting half the time $P_{\text {suc }}=1$ and half the time $P_{\text {suc }}=0.1$. Through which link must the packet be sent? If 
the conventional ETX metric is used, link $b$ is chosen because it presents $E\left[P_{\text {suc }}\right]=0.55$. Nevertheless, while link $b$ is in its worst condition, its average number of required transmissions is 10 . Thus, in a long term analysis, the average number of required transmissions is 5.5. Moreover, actual MAC layers present a limit to the number of retransmissions allowed. For the IEEE 802.11 standard, it is 7 when the RTS/CTS handshake is used. Therefore, link $b$ would, half the time, present a lossy condition, in which packets will be frequently discarded due to the retransmission limit. In order to avoid this kind of problem, our proposed metric takes into account the maximum number of MAC-layer retransmissions to select the routes. In the next section, we define the Average Number of Transmissions (ANT) function, which is used to calculate our proposed metric.

\section{A. The Average Number of Transmissions}

The ANT function represents the expected number of retransmissions on a link taking into account the maximum number of retransmissions allowed by the MAC-layer (MaxRetry). Before defining the ANT function we will introduce the concept of MAC-layer outage. We call MAC-layer outage the condition that arrives when the current Success Probability $\left(P_{S u c}\right)$ of a link results in an expected number of retransmissions higher than MaxRetry. In this situation, there is a high probability that the transmitted packet will be discarded due to an excessive number of retransmissions. The MAClayer outage occurs when the success probability of the link is smaller than the Limit Success Probability $\left(P_{\text {limit }}\right)$, which is defined as

$$
P_{\text {limit }}=\frac{1}{\text { MaxRetry }} .
$$

The ANT function is then defined as

$$
A N T(x)= \begin{cases}\frac{1}{P_{S u c}(x)} & P_{S u c}(x)>P_{\text {limit }} \\ \frac{1}{P_{\text {limit }}} & P_{\text {Suc }}(x) \leq P_{\text {limit }} .\end{cases}
$$

The ANT function behavior is depicted on Fig. 3.

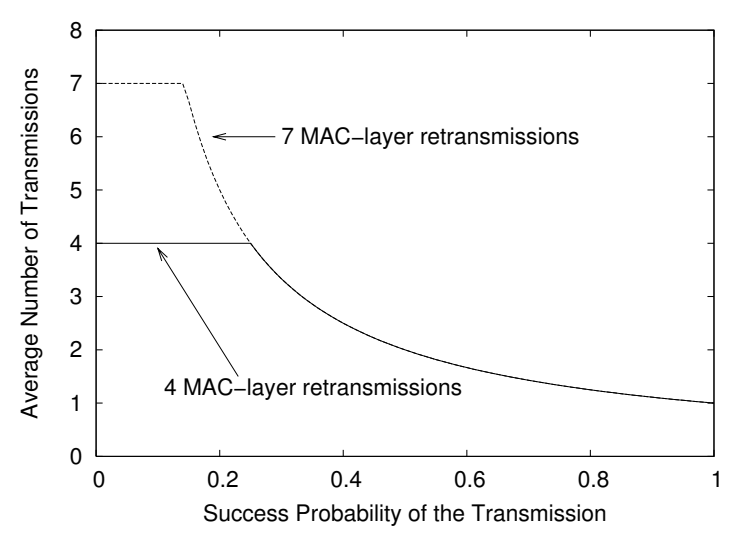

Fig. 3. Behavior of the ANT function.

As we observe on Fig. 3, the parameter MaxRetry limits the value of the ANT function. This limitation reflects that the MAC layer will not retransmit a packet more than MaxRetry times. The probability that the link will present a success probability lower than $P_{\text {limit }}$ is the MAC-layer outage probability $\left(P_{\text {out }_{M A C}}\right)$ of the link. Fig. 4 shows the outage probabilities of different links as a function of $E\left[P_{S u c}\right]$ assuming the 64-QAM modulation and 500-byte packets .

Fig. 4 shows that links with the same average $P_{S u c}$ may present different MAC-layer outage probabilities, depending on their K-factor. For the same $E\left[P_{S u c}\right]$, channels with more energy concentrated on the direct path present a smaller MAClayer outage probability. This effect is more pronounced when the MAC layer permits more retransmissions (Fig. 4(b)) because the higher MaxRetry parameter is, the lower the required $P_{S u c}$ to generate a MAC-layer outage. Thus, it is less probable that the success probability of channels with a strong line-ofsight will get below $P_{\text {limit }}$. The different behaviors of the average $P_{S u c}$ and $P_{\text {out }}{ }_{M A C}$ is the main incentive to the use of a more complete view of the wireless link to derive the routing metric. The following section formalizes the calculation of the DBETX metric and discusses its requirements.

\section{B. The Distribution Based Expected Transmission Count}

As discussed in the previous sections, the proposed metric has three main goals: (i) to reflect the variations of the wireless channel; (ii) to take into account the MAC layer retransmission limit, and (iii) to favor links with a lower loss probability. Thus, we define de DBETX metric for link $l$ as

$$
\operatorname{DBETX}(l)=E[A N T](l) * \frac{1}{1-P_{\text {out }_{M A C}}(l)} .
$$

By using the average ANT, we achieve our first two goals and by weighting the metric based on the MAC-layer outage probability, we arrive to our third goal. The calculation of the DBETX requires the knowledge of the actual behavior of the wireless channel instead of only knowing the average behavior of the channel. The main problem is the difference between the working time scales of the different layers. While the events of interest occur at the physical level in a granularity of milli or microseconds, network level interactions are explicitly reduced in order to reduce overhead and, as a consequence, occur at a time scale of seconds. Thus, it is impossible to have a complete view of the physical medium based only on the network level interactions.

We propose the use of passive overhearing to obtain the channel information for each received frame from a certain source node to update the information about the specific source-destination link. With this approach, after the reception of some frames, it is possible to estimate the probability density function of the received power of a link and the probability density function of the link SNIR.

According to the modulation scheme used, the appropriated function is used to translate SNIR into BER. From the BER, we obtain the PER for a given SNR as follows

$$
\operatorname{PER}(S N R)=1-[1-B E R(S N R)]^{n},
$$

where $n$ is the average packet length [13].

Finally, the DBETX metric is obtained from (5) and (6) by applying $P_{S u c}=1-P E R$. The entire path to obtain the DBETX is depicted in Fig. 5. 


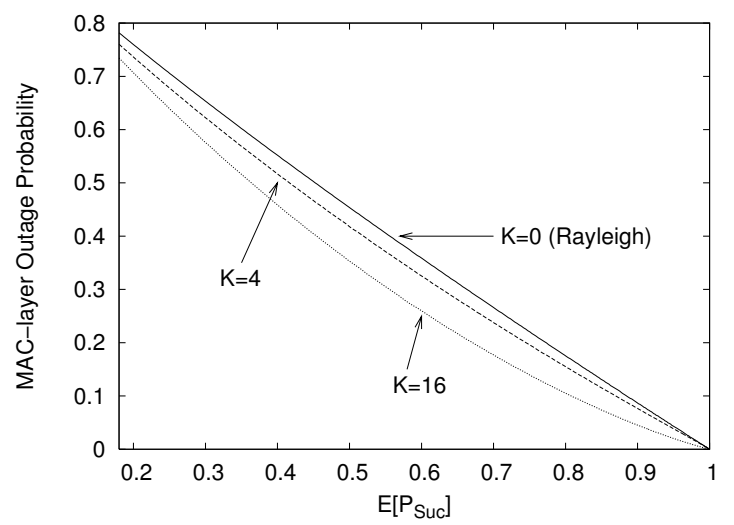

(a) Outage for 4 MAC-layer retries.

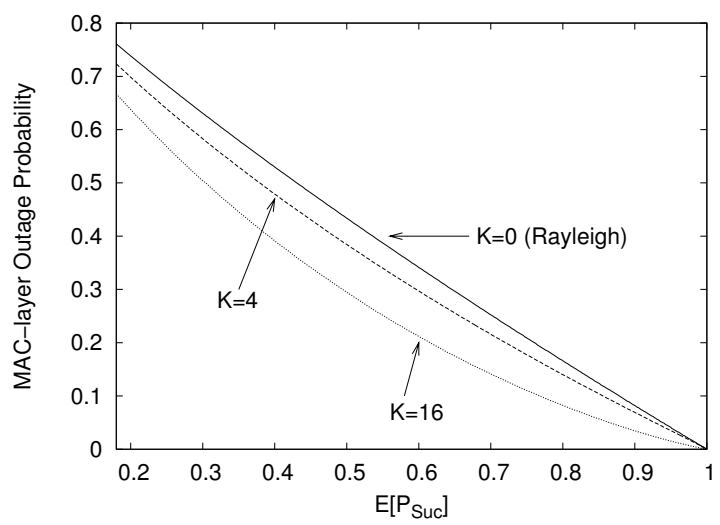

(b) Outage for 7 MAC-layer retries.

Fig. 4. MAC-layer outage probability as a function of the average success probability.

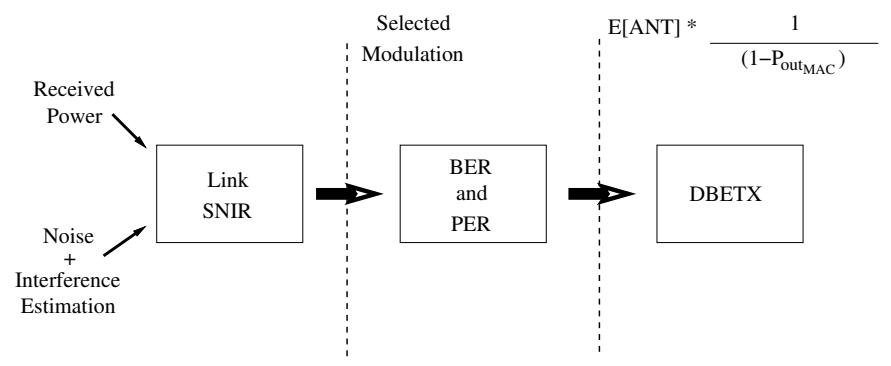

Fig. 5. Calculation of the DBETX metric.

In order to take into account the effect of the fading, each obtained value is weighted by the probability of the originating SNIR, which is obtained in the pdf estimation. In other words, $E[A N T]$ for link $l$ is obtained as

$$
E[A N T](l)=\sum_{S N I R=0}^{\infty} \operatorname{prob}(S N I R) * A N T(S N I R),
$$

where $\operatorname{prob}(S N I R)$ is the probability that the link $l$ will present the given SNIR.

We defined the DBETX metric based on the probability of success of a one-way transmission. Although the transmission of a packet usually involves the transmission of one or more control frames, we assume that these frames are transmitted in lower rates, whose error probability is negligible compared to the data rate error probability. In cases where this assumption does not hold, the extension of the model is straightforward. The next section details the performed simulations and discusses the results.

\section{Simulations}

In order to evaluate the efficiency of the proposed metric, we performed simulations of a multi-hop wireless network and compared the results of the DBETX to the results of the conventional ETX metric. We assume nodes are capable of correctly estimating the pdf of the experienced SNR and calculate the DBETX and ETX metric for each link based on this estimation.
Then, we use the Dijkstra algorithm to compute the routes from each node to every other node in the network. The cost of each route is computed using both routing metrics, ETX and DBETX. Finally, based on the computed routes, we calculate three distinct metrics: the average number of hops per route, the average availability per used link (defined as $1-P_{\text {out }_{M A C}}$ ), and the average number of transmissions (ANT) per used link.

The simulations were performed using the GNU Octave tool. The results are presented with a $95 \%$ confidence level and the confidence intervals are presented as vertical bars in the graphs.

\section{A. Simulation Scenario}

In order to create more realistic scenarios, whenever possible we based our configurations on the IEEE $802.11 \mathrm{~g}$ standard. The nodes transmission range $(R)$ is defined based on the characteristics of typical IEEE $802.11 \mathrm{~g}$ devices [11]. The simulation area is a rectangular surface with sides $\pi R$ and $6 R$. So, the total surface is $6 \pi R^{2}$. We measure the network density as a function of nodes per transmission area (a $\pi R^{2}$ area). The node density is varied through the simulations in order to evaluate its effects on the analyzed metrics.

The average received power is calculated using the logdistance propagation model with $\beta=2.7$, simulating a typical urban scenario [9]. The nodes are static and the signal fluctuations are assumed to result from environmental changes. The carrier frequency is set to $2.4 \mathrm{GHz}$. Moreover, we considered the 64-QAM as the modulation scheme. This is the modulation used in the highest data rate offered by the IEEE 802.11g and the BER is calculated as in [14]. The noise plus interference floor is assumed constant and configured to result in $P_{S u c}=10^{-5}$ at a distance $R$ in the absence of fading. The parameter MaxRetry is set to 7 . We set the average packet length to 500 bytes. Finally, to vary the type of channel, the value of the Ricean factor, $\mathrm{K}$, of each link is randomly picked in the set $\left[\begin{array}{llllllll}0 & 1 & 2 & 4 & 8 & 16 & 32 & 64\end{array}\right]$. Table I summarizes these parameters. 
TABLE I

Simulation PARAMETERS

\begin{tabular}{|l|c|}
\hline \multicolumn{2}{|c|}{ Used Values } \\
\hline \hline Transmission range $(\mathrm{R})$ & $39 \mathrm{~m}$ \\
\hline Simulation area & $\pi R \times 6 R \mathrm{~m}^{2}$ \\
\hline Attenuation factor $(\beta)$ & 2.7 \\
\hline Carrier frequency & $2.4 \mathrm{GHz}$ \\
\hline Modulation & $64-\mathrm{QAM}$ \\
\hline MaxRetry & 7 \\
\hline packet length & 500 bytes \\
\hline Ricean factor $(\mathrm{K})$ & 01248163264 \\
\hline
\end{tabular}

\section{B. Results}

The first analyzed metric is the average number of hops per route. Fig. 6 shows the average number of hops as a function of the network density.

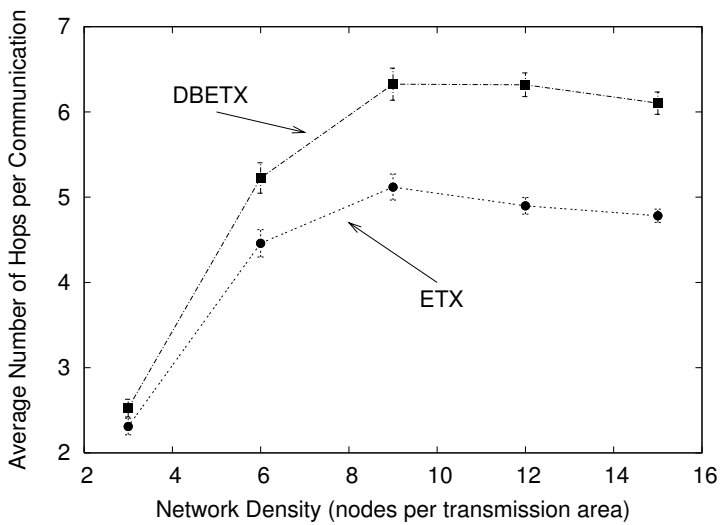

Fig. 6. Average number of hops per communication.

Analyzing Fig. 6, the first remark is that for very low network densities, the average number of hops tends to be very small. This is due to the low connectivity, which reduces the probability of a fully connected network. Thus, for low densities, nodes tend to be clustered. This clustering results in shorter routes. As the node density increases, the average number of hops also increases for the network tends to be more connected. Besides, the DBETX metric results in routes with more hops because DBETX trades more aggressively than the ETX metric distance for quality, choosing longer routes. Finally, the results with both ETX and DBETX present a behavior characterized by an initial increase followed by a reduction of the number of hops as the density increases. That is because the probability of finding reasonable longer hops is higher for higher densities, allowing a reduction on the route length.

Next, we analyze the Average Number of Transmissions per link. The lower this value, the best because a lower ANT means less wasted time and, thus, better resource utilization. Fig. 7 presents the results.

For low densities, the Average Number of Transmissions per link is high since nodes are forced to use bad quality links. With low densities, bad links without an alternate route becomes usual. Moreover, with fewer nodes, the average distance between neighbor nodes increases. Longer distances results in a lower average SNIR. As the density increases, both

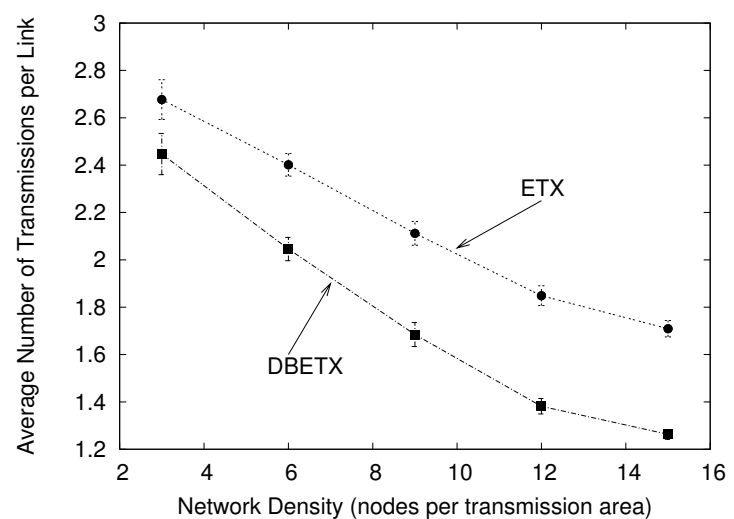

Fig. 7. Average number of transmissions per link.

metrics result in lower Average Number of Transmissions. The higher density reduces the average node distance, resulting in higher average SNIR, and creates more route choices, allowing the nodes to trade, for instance, a bad link for two good links.

It is worth noting that the improvement of the DBETX over the ETX in terms of Average Number of Transmissions increases with the node density. For a very low density of 3 nodes per transmission area, DBETX chooses links with an ANT more than $8 \%$ lower. As the density increases, more significant reduction in the Average Number of Transmissions is achieved. For an intermediate density of 9 nodes per transmission area, the reduction is already significant and the DBETX results in an ANT approximately 20\% lower. For a density of 15 nodes per transmission area, the reduction is close to $26 \%$.

We analyze now the average availability of the selected links. Higher availability values are good for they reflect more stable communications with lower loss probabilities and fewer interruptions. Fig. 8 shows the results.

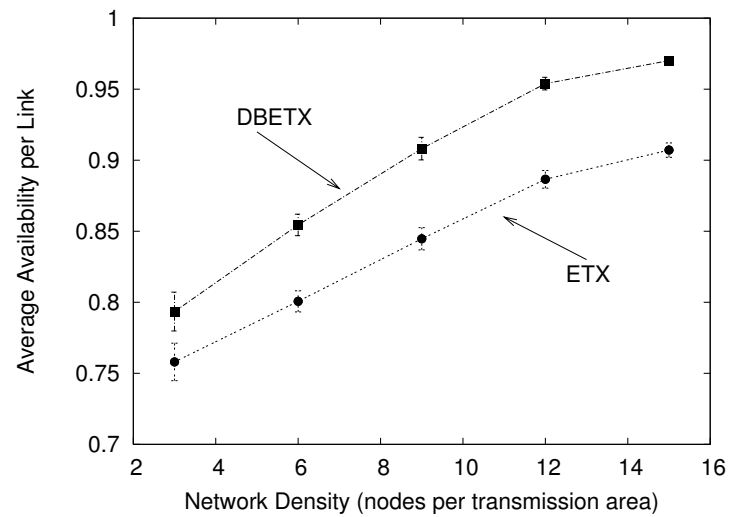

Fig. 8. Average availability per link.

In Fig. 8, we observe that the availability grows with the density. This is a consequence of the increased routing alternatives, allowing nodes to choose more stable routes. For very low densities, the average link availability achieved by ETX is around 0.76 and DBETX results in an average availability $5 \%$ higher. The reduced network connectivity forces nodes to use some bad links in order to reach otherwise 
inaccessible neighbors. As the density increases, both metrics use links with higher availability. The average availability achieved by DBETX is always higher than that obtained by ETX. Moreover, for a network density of 15 nodes per communication range, the average availability of the links used by DBETX is around 0.97 , and it is approximately $7 \%$ higher than the average availability obtained by ETX. This result confirms the attempt of the DBETX metric to avoid links based on their worst case condition.

Finally, based on Figs. 6 and 8 we estimate the end-to-end availability of the selected routes as Avail ${ }^{\text {Hops }}$, where Avail is the average availability per link, showed on Fig. 8, and Hops is the average number of hops per route, depicted on Fig. 6. The end-to-end availability reflects the probability that all the links of a route will be out of MAC-layer outage at the same time. Therefore, it reflects the probability that the chosen route will present low loss rate. Fig. 9 shows the results.

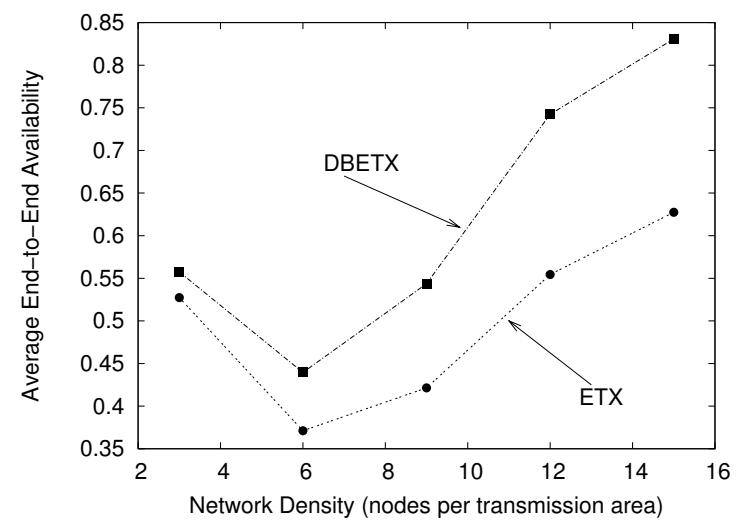

Fig. 9. End-to-End success probability.

Fig. 9 shows that the DBETX metric always results in a higher end-to-end availability than the ETX metric. Moreover, the improvement increases with the network density. It is worth noting that for very low density networks the average end-to-end availability is relatively high because in this scenario the network tends to be partitioned and the average number of hops is small. This explains why the density of 6 nodes per transmission area results in worst results than the density of 3 nodes per transmission area. When the density is equal to 6 nodes per transmission area, the network is more connected and the routes are longer. The improvement of DBETX over ETX is already $18 \%$ when the density is equal to 6 nodes per transmission area and it reaches $32 \%$ when the density is 15 nodes per transmission area.

\section{CONCLUSION}

In this paper we propose the use of more complete channel model to enhance the routing efficiency in wireless networks. We propose the Distribution Based Expected Transmission Count (DBETX) metric, which is based on the behavior of the perceived SNIR of a link. The DBETX is obtained using only local measurements and locally available information. Using cross-layer optimizations, the DBETX improves the network performance in the presence of fading channels.
The different link characteristics may result in very different SNIR profiles. The use of the DBETX, compared to the conventional ETX, avoids the selection of links whose worst case situation can dominate the link cost. The DBETX metric outperforms the conventional ETX metric, and its improvement increases with the network density. As density increases, the network becomes more connected, the average distances become smaller, and more routing choices are available. Thus, the improvement of DBETX over ETX becomes more significant. The proposed metric also reduces the average number of transmissions per link as compared to the conventional ETX metric. This is a direct consequence of avoiding links based on their worst case. Even for low density networks the improvement of DBETX is non-negligible and when the density is 15 nodes per transmission area DBETX results in approximately $26 \%$ less retransmissions per link. Moreover, results show that the proposed metric tends to use links presenting higher availability. This results in a higher endto-end availability and, as a consequence, a lower end-to-end loss probability.

As future works, we intend to analyze the effects of imperfect channel estimations in the DBETX metric and the number of samples needed to correctly estimate the probability density function of the received power. Moreover, we intend to extend our work to multi-rate networks.

\section{REFERENCES}

[1] M. E. M. Campista, I. M. Moraes, P. M. Espósito, A. Amodei Jr, D. O. Cunha, L. H. Costa, and O. C. M. B. Duarte, "The ad hoc return channel: a low-cost solution for brazilian interactive digital tv," IEEE Communications Magazine, vol. 45, no. 1, Jan. 2007.

[2] S. R. Das, C. E. Perkins, and E. M. Royer, "Performance comparison of two on-demand routing protocols for ad hoc networks," in IEEE INFOCOM, 2000

[3] R. Schmitz, M. Torrent-Moreno, H. Hartenstein, and W. Effelsberg, "The impact of wireless radio fluctuations on ad hoc network performance," in IEEE LCN, 2004.

[4] A. Adya, P. Bahl, J. Padhye, A. Wolman, and L. Zhou, "A multi-radio unification protocol for ieee 802.11 wireless networks," in BroadNets, 2004.

[5] D. D. Couto, D. Aguayo, J. Bicket, and R. Norris, "High-throughput path metric for multi-hop wireless routing," in MOBICOM, 2003.

[6] R. Draves, J. Padhye, and B. Zill, "Comparison of routing metrics for static multi-hop wireless networks," in SIGCOMM, 2004.

[7] M. Haeggi, "On routing in random rayleigh fading networks," IEEE Transactions on Wireless Communications, vol. 4, no. 4, July 2005.

[8] C. E. Koksal and H. Balakrishnan, "Quality aware routing in timevarying wireless mesh networks," IEEE Journal on Selected Areas in Communications, vol. 24, no. 11, Nov. 2006.

[9] T. S. Rappaport, Wireless Communications: Principles and Practice, 2nd ed. Prentice Hall PTR, 2001.

[10] J. Sanchez-Garcia and D. R. Smith, "Capture probability in ricean fading channels with power control in the transmitters," IEEE Transactions on Communications, vol. 50, no. 12, Dec. 2002.

[11] IEEE, "Wireless LAN medium access control (MAC) and physical layer (PHY) specifications: High-speed physical layer in the $2.4 \mathrm{GHz}$ band," IEEE Standard $802.11 \mathrm{~g}, 2003$.

[12] B. Sklar, "Rayleigh fading channels in mobile digital communication systems Part I: Characterization,” IEEE Communications Magazine, July 1997.

[13] Y. Y. Kim and S. qi Li, "Modeling multipath fading channel dynamics for packet data performance analysis," Wireless Networks, vol. 6, no. 6, Dec. 2000.

[14] D. Yoon, K. Cho, and J. Lee, "Bit error probability of m-ary quadrature amplitude modulation," in IEEE VTS-Fall VTC, 2000. 